\title{
Ruanjian Sanjie decoction exhibits antitumor activity by inducing cell apoptosis in breast cancer
}

\author{
XIUMEI ZHAO ${ }^{1 *}$, JING ZHAO ${ }^{2 *}$, RENJIE HU ${ }^{1}$, QIANG YAO ${ }^{3}$, GUIXIAN ZHANG ${ }^{1}$, \\ HONGSHENG SHEN $^{1}$, ERNESTO YAGÜE ${ }^{4}$ and YUNHUI HU ${ }^{2}$
}

\begin{abstract}
${ }^{1}$ Centre for Research and Development of Anti Tumor Drugs, Tianjin Institute of Medical and Pharmaceutical Sciences, Tianjin 300020; ${ }^{2}$ The Third Department of Breast Cancer, Tianjin Medical University Cancer Institute and Hospital, Tianjin 300060; ${ }^{3}$ Tianjin People's Hospital, Tianjin 300121, P.R. China; ${ }^{4}$ Cancer Research Center, Division of Cancer, Faculty of Medicine, Imperial College London, Hammersmith Hospital Campus, London W12 0NN, UK
\end{abstract}

Received June 29, 2016; Accepted January 6, 2017

DOI: $10.3892 / 01.2017 .5832$

\begin{abstract}
Traditional Chinese medicine, based on theories developed and practiced for $>2,000$ years, is one of the most common complementary and alternative types of medicine currently used in the treatment of patients with breast cancer. Ruanjian Sanjie (RJSJ) decoction, is composed of four herbs, including Ban xia (Pinellia ternata), Xia ku cao (Prunella vulgaris), Shan ci gu (Cremastra appendiculata) and Hai zao (Sargassum pallidum), and has traditionally been used for softening hard lumps and resolving hard tissue masses. However, the active compounds and mechanisms of action of RJSJ remain unknown. The present study demonstrated the antitumor activity of RJSJ against Ehrlich ascites carcinoma in Swiss albino mice and breast cancer xenografts in nude mice. Notably, RJSJ does not induce body weight loss, immune function toxicity or myelosuppression in mice, indicating that it is safe and well tolerated. In addition, RJSJ shows potent cytotoxicity against breast cancer cells in vitro by the suppression of the anti-apoptotic proteins B-cell lymphoma 2 and survivin, leading to the activation of caspase-3/7 and caspase-9, and the apoptotic cascade. These findings provide a clear rationale to explore the therapeutic strategy of using RJSJ alone or in combination with chemotherapeutic agents
\end{abstract}

Correspondence to: Dr Yunhui Hu, The Third Department of Breast Cancer, Tianjin Medical University Cancer Institute and Hospital, Huan-Hu-Xi Road, Ti-Yuan-Bei, Hexi, Tianjin 300060, P.R. China

E-mail: yunhuihu200408@163.com

Dr Ernesto Yagüe, Cancer Research Center, Division of Cancer, Faculty of Medicine, Imperial College London, Hammersmith Hospital Campus, London W12 0NN, UK

E-mail: ernesto.yague@imperial.ac.uk

*Contributed equally

Key words: Ruanjian Sanjie decoction, breast cancer, antitumor, apoptosis, Ehrlich ascites carcinoma for breast cancer patients and the characterization of its active principles.

\section{Introduction}

Breast cancer is the most frequent type of malignancy among women worldwide (1). Although current treatments are often associated with excellent short-term prognoses, $\leq 13 \%$ of women develop recurrence within 9 years of the initial treatment. In addition, $>60 \%$ of women with localized breast cancer eventually develop distant, late-stage disease (2). In China, $>20 \%$ of patients with breast cancer are diagnosed at an advance stage of the disease, typically with metastatic invasion (3). Although a large number of successful treatment options are available for early breast cancer, standard treatment protocols for advanced breast cancer cannot currently circumvent patient relapse (4). Genetic reprogramming during the metastatic process enables cancer cells to invade distant organs and to acquire resistance to numerous types of chemo- and radiotherapy, contributing to the high mortality of advanced-stage breast cancer (5). Thus, improved therapeutic options for the affected patients are required.

Complementary therapies such as traditional Chinese medicine (TCM) have increased in popularity as a less intensive and more 'natural' approach to achieving health or improving quality of life, particularly for patients with advanced-stage cancer (6). In the Yellow Emperor's Classic of Internal Medicine, written circa $250 \mathrm{BC}$, the first clinical description and phytotherapeutic treatment of breast cancer was recorded. Besides anecdotal evidence of a cure for breast cancer using TCM as a sole therapy (6), previous studies suggest that TCM may be effective in breast cancer management. For example, Huaier (Trametes robiniophila Murr) sensitizes breast cancer cells to radiotherapy through the regulation of cell cycle and DNA repair pathways (7). In vitro and in vivo testing of Scutellaria barbata in breast cancer has led to an ongoing clinical trial (8); and a recent population-based study suggests that adjunctive TCM therapy may lower the risk of mortality in patients with advanced breast cancer (9).

Ruanjian Sanjie (RJSJ), composed of Ban xia (Pinellia ternata), Xia ku cao (Prunella vulgaris), Shan ci gu 
(Cremastra appendiculata) and Hai zao (Sargassum pallidum), is an empirical traditional Chinese herbal decoction used for softening hard lumps and resolving hard masses (10). Although the active principles and the mechanisms of action remain unknown, extracts from certain individual herbal components of RJSJ exhibit anticancer properties. For example, Prunella vulgaris extracts exhibit growth-suppressive activity on gastric cancer (11) and migration-suppressive activity on liver cancer cells (12), whereas Sargassum pallidum extracts exhibit cytotoxic effects in breast cancer MCF-7 cells in vitro (13) and inhibit pancreatic cancer relapse in vivo (14). The present study demonstrates that RJSJ exhibits antitumor activity against Ehrlich ascites carcinoma (EAC) in Swiss albino mice and breast cancer xenografts in nude mice. RJSJ shows potent cytotoxicity against breast cancer cells in vitro by the suppression of the anti-apoptotic proteins B-cell lymphoma 2 (Bcl-2) and survivin, leading to the activation of caspase-3/7 and caspase- 9 , and the apoptotic cascade. The present findings offer a clear rationale to additionally explore the therapeutic strategy of using RJSJ alone or in combination with chemotherapeutic agents for patients with breast cancer and the characterization of its active principles.

\section{Materials and methods}

Herbs and preparation of aqueous extracts. All herbs in the RJSJ formula were obtained from Tianjin Zhong Xin Pharmaceutical Group Corporation Ltd. (Tianjin, China). The dry weights of the four herbs were at fixed ratios (Pinellia ternate: Prunella vulgaris: Cremastra appendiculata: Sargassum pallidum=3:3:2:2). Whole herbs $(900 \mathrm{~g})$ were ground to a powder and extracted twice with hot distilled water, first with 10.5 liters and next with 15 liters, at $100^{\circ} \mathrm{C}$ for $30 \mathrm{~min}$ each. The solutions were combined and centrifuged at $3,000 \mathrm{x} g$ for $10 \mathrm{~min}$ at room temperature. The resulting supernatant was filtered through $0.45-\mu \mathrm{m}$ cellulose acetate filters, concentrated using a rotary evaporator and spray-dried to obtain a brown fine powder. This residue was dissolved in PBS at the desired concentration and stored at $-20^{\circ} \mathrm{C}$ as the stock extract RJSJ solution.

Reagents, cell lines and mice. The breast cancer MDA-MB-231 and MCF-7 cell lines were obtained from the American Type Culture Collection (Manassas, VA, USA) through the Cell Resource Center of the Tianjin Cancer Hospital (Tianjin, China) and were authenticated by short tandem repeat profiling. The cells were cultured in Dulbecco's modified Eagle's medium (Invitrogen; Thermo Fisher Scientific, Inc., Waltham, MA, USA) with $10 \%$ fetal calf serum and $2 \mathrm{mM}$ L-glutamine (Gibco; Thermo Fisher Scientific, Inc.). The cultures were maintained at $37^{\circ} \mathrm{C}$ in an air- $5 \% \mathrm{CO}_{2}$ incubator at constant humidity. Doxorubicin, 5-flurouracil (5-Fu) and MTT were purchased from Sigma-Aldrich; Merck Millipore (Darmstadt, Germany). All animals, including 120 Swiss albino mice (60 female and 60 male; age, 5-6 weeks; weight, 25-30 g) and 24 female nude mice (age, 5-6 weeks; weight, 20-25 g) were obtained from Vital River Laboratories Co., Ltd. (Beijing, China) and used according to the National Institutes of Health guidelines for animal care (15). Animals were maintained at between 21 and $24^{\circ} \mathrm{C}$ with an $8 \mathrm{~h}$ light $/ 16 \mathrm{~h}$ dark cycle, and were allowed to feed ad libitum. All in vivo studies were performed in the Central Laboratory of the Tianjin Institute of Medical and Pharmaceutical Sciences (Tianjin, China), and approved by the Committee on Ethics of the Tianjin Institute of Medical and Pharmaceutical Sciences.

Acute toxicity study. An acute oral toxicity assay was performed using healthy, non-pregnant, adult female, Swiss albino mice (weight range, 25-30 g) divided into six groups. Increasing oral doses of RJSJ $(312.5,625,1,250,2,500$ and $5,000 \mathrm{mg} / \mathrm{kg}$ body weight) in distilled water were administered by intragastric (i.g.) administration at $20 \mathrm{ml} / \mathrm{kg}$ to the different test groups. The control group received distilled water only. Following treatment, the mice were allowed to feed ad libitum and monitored for mortality or any behavioral changes for 14 days (16).

EAC tumor model and RJSJ treatment. The EAC cells were obtained from Tianjin Institute of Medical and Pharmaceutical Sciences (Tianjin, China) and were maintained in vivo in the Swiss albino mice by intraperitoneal (i.p.) transplantation of $2 \times 10^{6}$ cells per mouse every 10 days. For treatments, mice were divided into four groups of 12 animals each as follows: Group 1, control group; group 2, treated every other day with $20 \mathrm{mg} / \mathrm{kg}$ 5-Fu by i.p. injection; group 3, treated daily with $500 \mathrm{mg} / \mathrm{kg}$ RJSJ by i.g. administration; and group 4 , treated with $20 \mathrm{mg} / \mathrm{kg}$ 5-Fu by i.p. injection combined with i.g. $500 \mathrm{mg} / \mathrm{kg}$ RJSJ. The experiment was started by inoculating EAC cells ( $2 \times 10^{6}$ cells per mouse) subcutaneously to all animals, and the treatments were initiated $24 \mathrm{~h}$ subsequently. On day 8 , subsequent to the administration of the last dose followed by $18 \mathrm{~h}$ fasting, all animals were sacrificed by cervical dislocation. Thymi and spleens were collected for thymus and spleen index determination calculated according to the following formulae: Spleen index=spleen weight $(\mathrm{mg}) /$ body weight $(\mathrm{g})$; and thymus index=thymus weight (mg)/body weight (g).

In vivo breast cancer xenografts. A total of $1 \times 10^{7}$ MDA-MB-231 cells were suspended in $0.1 \mathrm{ml}$ PBS containing $50 \%$ Matrigel (BD Biosciences, Franklin Lakes, NJ, USA) and injected into the mammary fat pad of 4-5-week-old female nude mice. Tumor size was measured every other day in two dimensions using a caliper, and the tumor volume was calculated with the following formula: Tumor volume $\left(\mathrm{mm}^{3}\right)=0.5 \mathrm{xab}^{2}$ (with a and $\mathrm{b}$ being the longest and shortest diameter of the tumor, respectively). When the average tumor volume reached $400 \mathrm{~mm}^{3}$, the tumor-bearing mice were randomly divided into four groups with 6 animals/group: Group 1, control group, received normal saline; group 2 was treated every other day with $1 \mathrm{mg} / \mathrm{kg}$ doxorubicin by i.p. injection; group 3 was treated daily with $500 \mathrm{mg} / \mathrm{kg}$ RJSJ by i.g. administration; and group 4 was treated with a combination of $1 \mathrm{mg} / \mathrm{kg}$ doxorubicin and $500 \mathrm{mg} / \mathrm{kg}$ RJSJ as aforementioned. Tumor volume was monitored until the mice were sacrificed by cervical dislocation when the tumor size was $>18 \mathrm{~mm}$ in diameter in either direction, and the tumors were collected for RNA extraction.

Cell viability analysis. MTT assays were performed to evaluate the cell viability in response to drug treatments and to 
determine the concentration of drug that inhibited cell growth by $50 \%\left(\mathrm{IC}_{50}\right)$ subsequent to 3 days of treatment (17).

Caspase activity measurement. A total of $1 \times 10^{4}$ cells were incubated with RJSJ in a 96-well plate for $24 \mathrm{~h}$. The caspase- $3 / 7$ and caspase- 9 activities were measured using the Caspase-Glo 3/7 Assay and Caspase-Glo 9 Assay kits, respectively (Promega Corporation, Madison, WI, USA), according to the manufacturer's protocol. Fluorescence was measured using a GloMax 20/20 Luminometer (Promega Corporation).

Hoechst 33258 staining assay. The nuclear morphology of the cells treated with RJSJ was observed subsequent to Hoechst 33258 staining. The MCF-7 and MDA-MB-231 cells, at a density of $2.5 \times 10^{5} /$ well, were seeded in 6-well plates, cultured for $24 \mathrm{~h}$, and treated with different concentrations of RJSJ for $48 \mathrm{~h}$. The cells were then washed twice with PBS, fixed in $4 \%$ paraformaldehyde at $4^{\circ} \mathrm{C}$ for $30 \mathrm{~min}$, and stained with $10 \mu \mathrm{g} / \mathrm{ml}$ Hoechst 33258 for $15 \mathrm{~min}$ at $37^{\circ} \mathrm{C}$. Changes in nuclear morphology were monitored with an Olympus IX51 inverted microscope (Olympus Corporation of the Americas, Inc., Central Valley, PA, USA).

Flow cytometry. For cell apoptosis analysis, an Annexin V-fluorescein isothiocyanate (FITC) and propidium iodide (PI) double-staining apoptosis detection kit (BD Biosciences) were used according to the procedure suggested by the manufacturer. In total, $2 \times 10^{5}$ cells were washed twice with PBS and suspended in $100 \mu \mathrm{l}$ binding buffer, followed by staining with $5 \mu \mathrm{l}$ Annexin V-FITC and $5 \mu \mathrm{l}$ PI for $15 \mathrm{~min}$ in the dark at room temperature. The fluorescence intensity was measured with a flow cytometer (BD FACSCanto II; BD Biosciences) and the average percentage of Annexin V-positive cells was used as a measure of apoptosis, both in the early and late stages, according to $\mathrm{Hu}$ et al (18).

Drug resistance clonogenic assay. A total of $1 \times 10^{5}$ cells/well in a 6-well plate were treated with different concentrations of RJSJ for 1 week. Resistant clones were fixed with $4 \%$ paraformaldehyde, stained with $0.2 \%$ crystal violet and counted. The crystal violet retained in the cells was quantified by solubilization with $0.5 \%$ acetic acid and measurement of optical density at $592 \mathrm{~nm}$.

RNA isolation and reverse transcription-quantitative polymerase chain reaction ( $R T-q P C R)$. Total RNA was isolated using TRIzol (Invitrogen; Thermo Fisher Scientific, Inc.) following the procedure suggested by the manufacturer. The complementary(c) DNA was generated using oligo(dT) primers and SuperScript III Reverse Transcriptase (Invitrogen; Thermo Fisher Scientific, Inc.) using $2 \mu \mathrm{g}$ total RNA. Specific primers for each gene (Table I) were designed using Primer Express software (version 3.0; Applied Biosystems; Thermo Fisher Scientific, Inc.). qPCR was carried out using SYBR-Green I (Takara Biotechnology Co., Ltd., Dalian, China) and detected using an ABI Prism ${ }^{\circledR}$ SDS 7900 Real-Time PCR System (Applied Biosystems; Thermo Fisher Scientific, Inc.). The cycling conditions were $95^{\circ} \mathrm{C}$ for $15 \mathrm{sec}$ and $60^{\circ} \mathrm{C}$ for $1 \mathrm{~min}$, for 40 cycles. A standard curve for each gene was included in each PCR amplification for the calculation of the
Table I. Oligonucleotides used for quantitative polymerase chain reaction.

\begin{tabular}{ll}
\hline Name & \multicolumn{1}{c}{ Sequence (5' to 3') } \\
\hline $\begin{array}{l}\text { RPS14-forward } \\
\text { RPS14-reverse }\end{array}$ & TCACCGCCCTACACATCAAACT \\
Bcl-2-forward & CAGTTGGGCAACAGAGAACCAT \\
Bcl-2-reverse & AGCCCTTGTCCCCAATTTGGAA \\
Survivin-forward & GGACCACCGCATCTCTACAT \\
Survivin-reverse & GACAGAAAGGAAAGCGCAAC \\
\hline
\end{tabular}

Bcl-2, B-cell lymphoma 2; RPS14, ribosomal protein S14.

Table II. Results of acute toxicity studies.

\begin{tabular}{lccccc}
\hline & \multicolumn{2}{c}{ Animals (n) } & & \multicolumn{2}{c}{ Body weight $(\mathrm{g})$} \\
\cline { 2 - 3 } \cline { 5 - 6 } Groups & Day 1 & Day 14 & & Day 1 & Day 14 \\
\hline Control & 12 & 12 & & $20.88 \pm 0.80$ & $30.96 \pm 2.04$ \\
RJSJ (mg/kg) & & & & & \\
312.5 & 12 & 12 & & $20.88 \pm 0.80$ & $28.96 \pm 2.52^{\mathrm{a}}$ \\
625 & 12 & 12 & & $20.88 \pm 0.80$ & $30.26 \pm 1.94^{\mathrm{a}}$ \\
1,250 & 12 & 12 & & $20.88 \pm 0.80$ & $29.57 \pm 2.26^{\mathrm{a}}$ \\
2,500 & 12 & 12 & & $20.88 \pm 0.80$ & $31.06 \pm 2.57^{\mathrm{a}}$ \\
5,000 & 12 & 12 & $20.88 \pm 0.80$ & $29.71 \pm 2.83^{\mathrm{a}}$ \\
\hline
\end{tabular}

Data are presented as the mean \pm standard deviation ( $\mathrm{n}=12$ per group). ${ }^{a} \mathrm{P}>0.05$ vs. control group. RJSJ, Ruanjian Sanjie.

quantification cycle value (19). Relative transcript levels were normalized with ribosomal protein S14.

Protein extraction and western blotting. A modified radioimmunoprecipitation assay buffer $(50 \mathrm{mM}$ Tris- $\mathrm{HCl}, 150 \mathrm{mM}$ $\mathrm{NaCl}, 0.25 \%$ SDS, $1 \%$ Triton $\mathrm{X}-100,0.25 \%$ sodium deoxycholate, $1 \mathrm{mM}$ EDTA, $1 \mathrm{mM}$ ethylene glycol-bis ( $\beta$-aminoethyl ether)-N,N,N',N'-tetraacetic acid and $1 \mathrm{mM}$ dithiothreitol) with a mixture of protease inhibitors (EMD Millipore, Billerica, MA, USA) was used for protein isolation from whole cells or nuclear fraction. Protein concentrations were determined using a bicinchoninic acid protein assay kit (Pierce; Thermo Fisher Scientific, Inc.). An equal quantity, $50 \mu \mathrm{g}$, of protein was resolved on $12 \%$ polyacrylamide gels and transferred onto nitrocellulose membranes (EMD Millipore), and blocked with 5\% blotting-grade milk (Bio-Rad Laboratories, Inc., Hercules, CA, USA) in PBS containing 0.1\% Tween-20. The membranes were incubated with primary antibodies against Bcl-2 (1:1,000 dilution; cat. no., 50E3; Cell Signaling Technology Inc., Danvers, MA, USA) or survivin (1:1,000 dilution; cat. no. 71G4B7; Cell Signaling Technology, Inc.) at $4^{\circ} \mathrm{C}$ overnight. A horseradish peroxidase-conjugated anti-rabbit immunoglobulin G secondary antibody (1:2,000 dilution; cat. no. 7074; Cell Signaling Technology, Inc.) was incubated with the membranes for $2 \mathrm{~h}$ at room temperature. Immunoblotting 
A

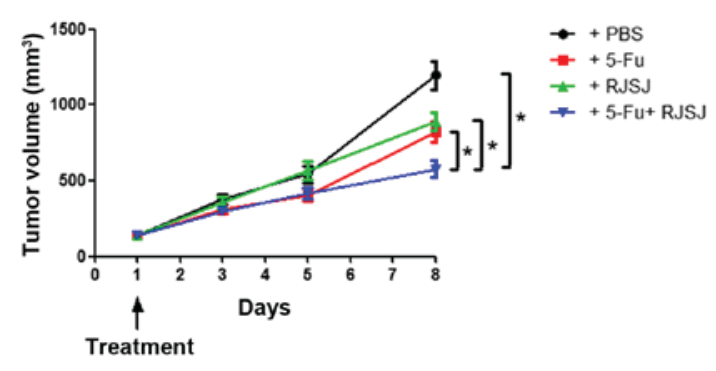

C
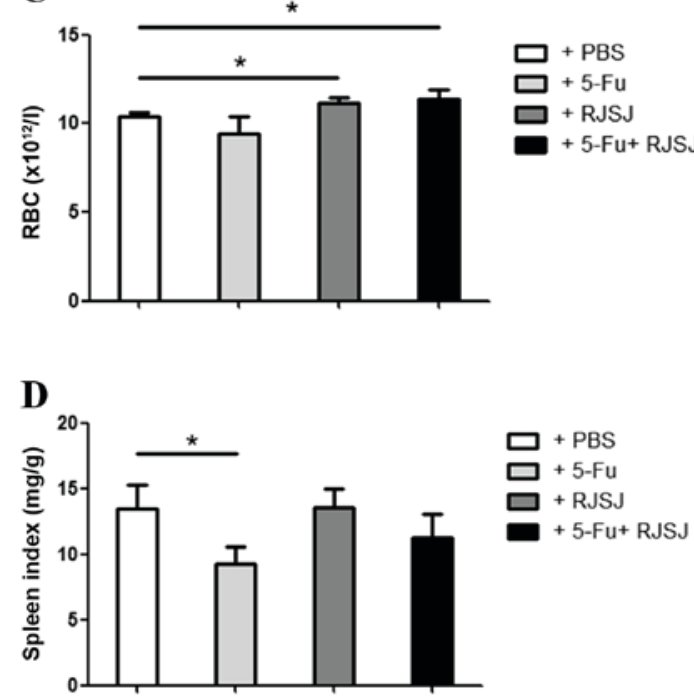

$\mathbf{E}$

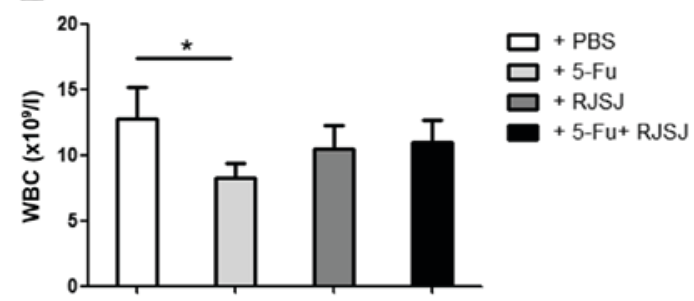

B
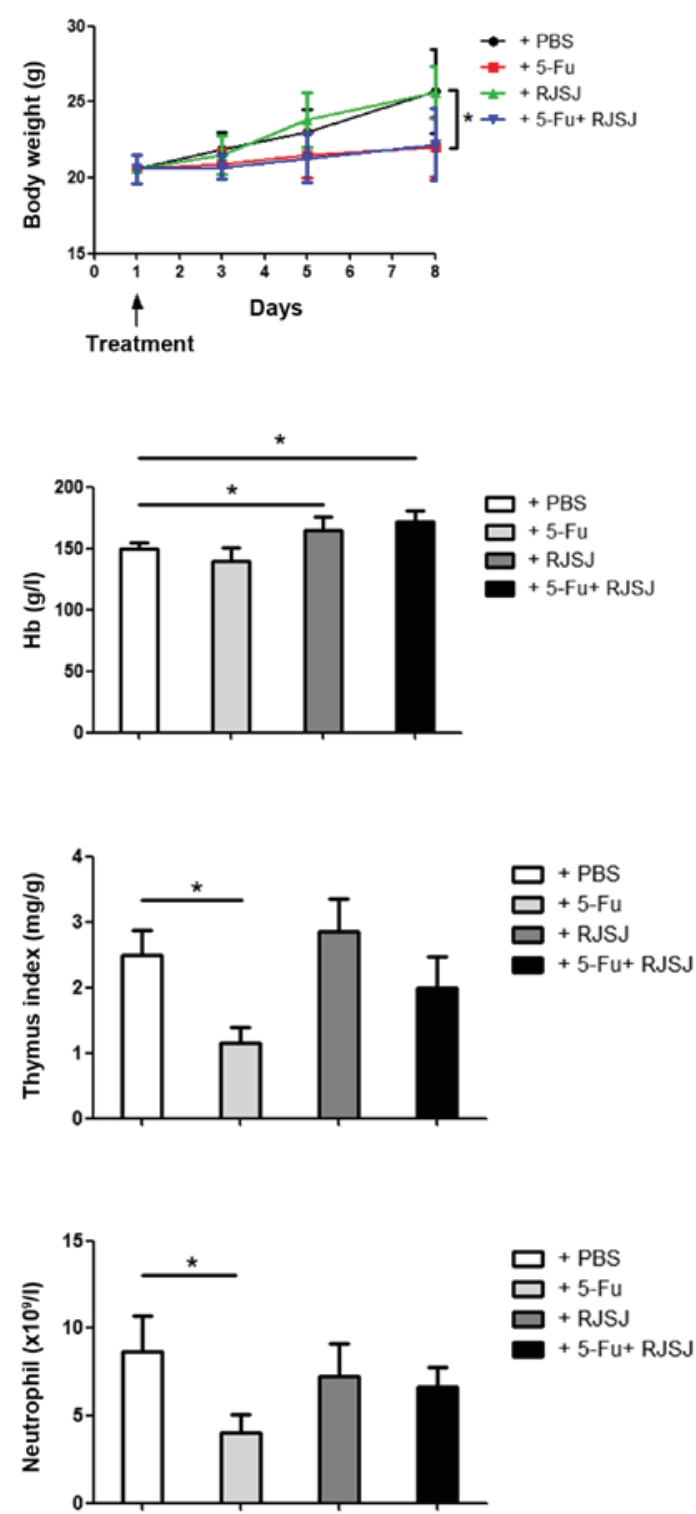

Figure 1. RJSJ treatment is effective and well tolerated in EAC tumor models. (A) Tumor size and (B) body weight of EAC-bearing mice subsequent to treatment with PBS (control), 5-Fu, RJSJ or 5-Fu plus RJSJ. (C) Effect of different drug treatments on RBC count, left panel, and Hb content, right panel. (D) Effect of different drug treatments on the spleen, left panel, and thymus, right panel, indexes. (E) Effect of different drug treatments on WBC, left panel, and neutrophil, right panel, counts. The results presented are the mean \pm standard deviation of 12 mice per group. ${ }^{*}<0.05$. RBC, red blood cell; WBC, white blood cell; RJSJ, Ruanjian Sanjie; EAC, Ehrlich ascites carcinoma; Hb, hemoglobin; 5-Fu, 5-fluorouracil.

signals were detected using the SuperSignal West Pico Chemiluminescent Substrate (Pierce; Thermo Fisher Scientific, Inc.), according to the manufacturer's protocol. All the membranes were re-probed with anti- $\beta$-actin antibody (1:200 dilution; cat. no. sc47778; Santa Cruz Biotechnology, Inc., Dallas, TX, USA), which served as a loading control.

Statistical analysis. All statistical analyses were performed using SPSS software (version 22.0; IBM SPSS, Armonk, NY, USA). All in vitro experiments were performed in triplicate and the results are presented as the mean \pm standard deviation. Analysis of variance followed by a Tukey's test was performed in the present study to evaluate significant differences among groups. Two-sided $\mathrm{P}<0.05$ was considered to indicate a statistically significant difference.

\section{Results}

Acute toxicity studies. In order to evaluate the potential RJSJ toxicity, the present study performed an acute toxicity study. For this purpose, the mortality of animals treated with the highest RJSJ dose $(\leq 5,000 \mathrm{mg} / \mathrm{kg})$ was monitored. The present study observed that RJSJ was safe at oral doses as high as $5,000 \mathrm{mg} / \mathrm{kg}$ body weight, causing no mortality, behavioral change, locomotor ataxia, diarrhea or weight loss in mice during 14 days of observation. Additionally, food and water intake did not differ between the groups studied (Table II).

Administration of RJSJ in combination with 5-Fu is more effective and safer than the chemotherapeutic treatment alone in EAC tumor models. To test the RJSJ antitumor efficacy 
A

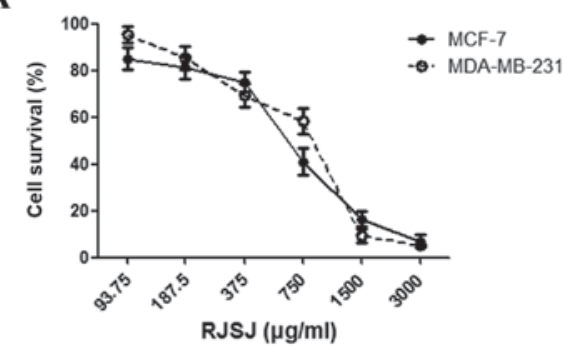

B

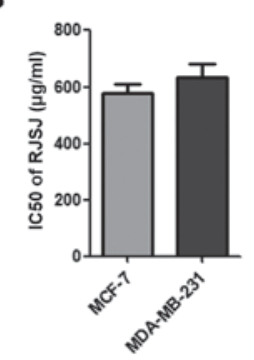

C
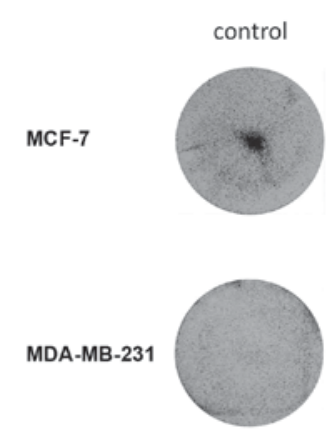
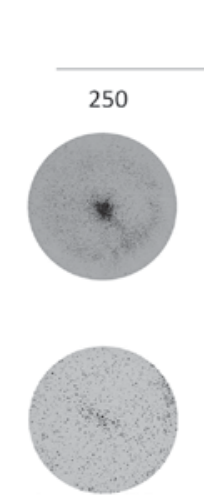

$+\operatorname{RJSJ}(\mu \mathrm{g} / \mathrm{ml})$
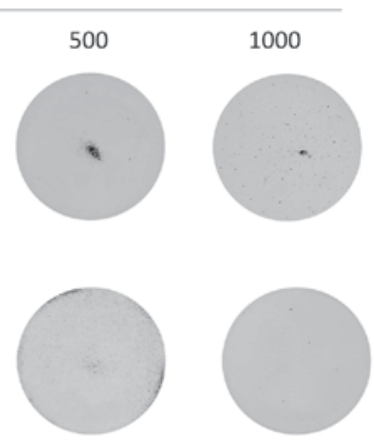
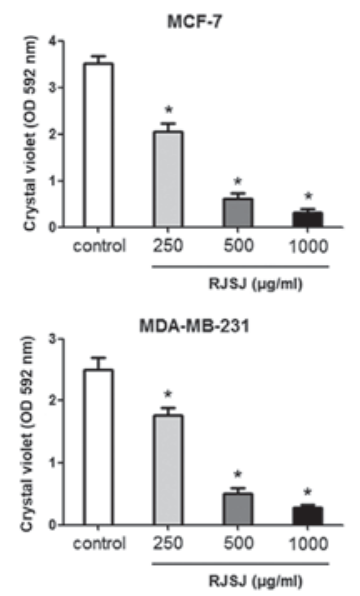

Figure 2. RJSJ is cytotoxic to breast cancer cells. (A) Dose-response curves used to calculate the $\mathrm{IC}_{50}$ of RJSJ for MCF-7 and MDA-MB-231 cells. (B) IC I0 values of RJSJ of MCF-7 and MDA-MB-231 cells. (C) Cells were treated with different concentrations of RJSJ in 6-well dishes for 7 days and then stained with crystal violet. Pictorial data are representative images of wells from 3 independent replicates, left panel. The dye was solubilized and the OD at 592 nm was measured, right panel. Numerical data represent the mean \pm standard deviation of 3 independent experiments. * $\mathrm{P}<0.05$. RJSJ, Ruanjian Sanjie; $\mathrm{IC}_{50}, 50 \%$ inhibitory concentration; OD, optical density.

in vivo, EAC tumor models were established. The animals were divided into different groups and treated with 5-Fu, RJSJ or 5-Fu plus RJSJ. Tumor growth steadily progressed during the following 8 days in the control group, whereas tumor volume increased at similar rates in the 5-Fu and RJSJ groups $(\mathrm{P}=0.86)$. The animals receiving $5-\mathrm{Fu}$ alone exhibited significant body weight loss $(\mathrm{P}=0.037)$, which was absent in those animals treated with RJSJ alone. Notably, the group receiving 5-Fu and RJSJ exhibited the slowest tumor growth, with body weight loss not greater than that obtained with $5-\mathrm{Fu}$ alone (Fig. 1A and B).

The hematological parameters of mice treated with RJSJ were observed to be significantly different to those from 5-Fu-treated mice. Although there were no differences in the hemoglobin content or red blood cell count between control and 5-Fu-treated mice $(\mathrm{P}=0.73)$, treatment with RJSJ at a dose of $500 \mathrm{mg} / \mathrm{kg}$ body weight significantly increased the level of the aforementioned hematological parameters (Fig. 1C) $(\mathrm{P}=0.04)$. As the thymus and spleen are organs directly affecting the immune function, the present study determined the effect of the aforementioned treatments on the thymus and spleen indexes. As expected from a chemotherapeutic drug, 5 -Fu affected the two organs, with a significant reduction in both thymus and spleen indexes $(\mathrm{P}=0.02)$. However, RJSJ blocked the side effects of 5-Fu, and the thymus and spleen indexes were indistinguishable between the combination and control groups (Fig. 1D) ( $\mathrm{P}=0.92$ and 0.95, respectively). Additionally, the white blood cell and neutrophil counts declined in the 5-Fu treatment group, a toxic side effect due to myelosuppression (20). By contrast, RJSJ in combination with 5-Fu prevented these side effects (Fig. 1E). In summary, treatment with RJSJ at $500 \mathrm{mg} / \mathrm{kg}$ exhibited significant anticancer activity; however, the administration of RJSJ together with 5-Fu exhibited a greater effect in inhibiting tumor growth than either compound alone without inducing body weight loss or reducing the level of immune function or myelosuppression. Thus, the administration of RJSJ in combination with 5-Fu is more effective and safer than the chemotherapeutic treatment alone.

Toxicity of RJSJ against breast cancer cells. As TCM has been used in the management of breast cancer (6-9), the present study monitored the effect of RJSJ on the growth of breast cancer MCF-7 and MDA-MB-231 cell lines using MTT assays. RJSJ exhibited an inhibitory effect on the growth of the cell lines, with $\mathrm{IC}_{50}$ values of $578 \mu \mathrm{g} / \mathrm{ml}$ for MCF-7 and $635 \mu \mathrm{g} / \mathrm{ml}$ for MDA-MB-231 (Fig. $2 \mathrm{~A}$ and B). At $1 \mathrm{mg} / \mathrm{ml}$, RJSJ showd cytotoxicity on $80-90 \%$ of the cancer cells. To additionally evaluate the cytotoxic effect of RJSJ on breast cancer cells, MCF-7 and MDA-MB-231 cells were treated with RJSJ, and crystal violet staining was used to determine the cell mass 1 week subsequent to treatment. Consistent with the aforementioned MTT assays, RJSJ significantly decreased the capacity of breast cancer cells to survive in a dose-dependent manner (Fig. 2C) $(\mathrm{P}=0.02)$. Thus, RJSJ is a potent cytotoxic agent against breast cancer cells. 
A
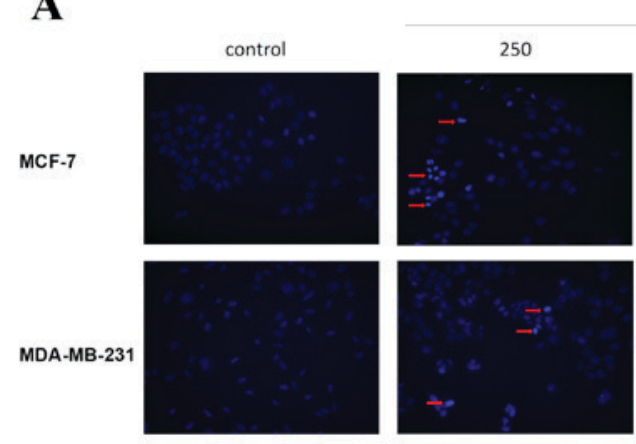

$+\operatorname{RJSJ}(\mu \mathrm{g} / \mathrm{ml})$

B
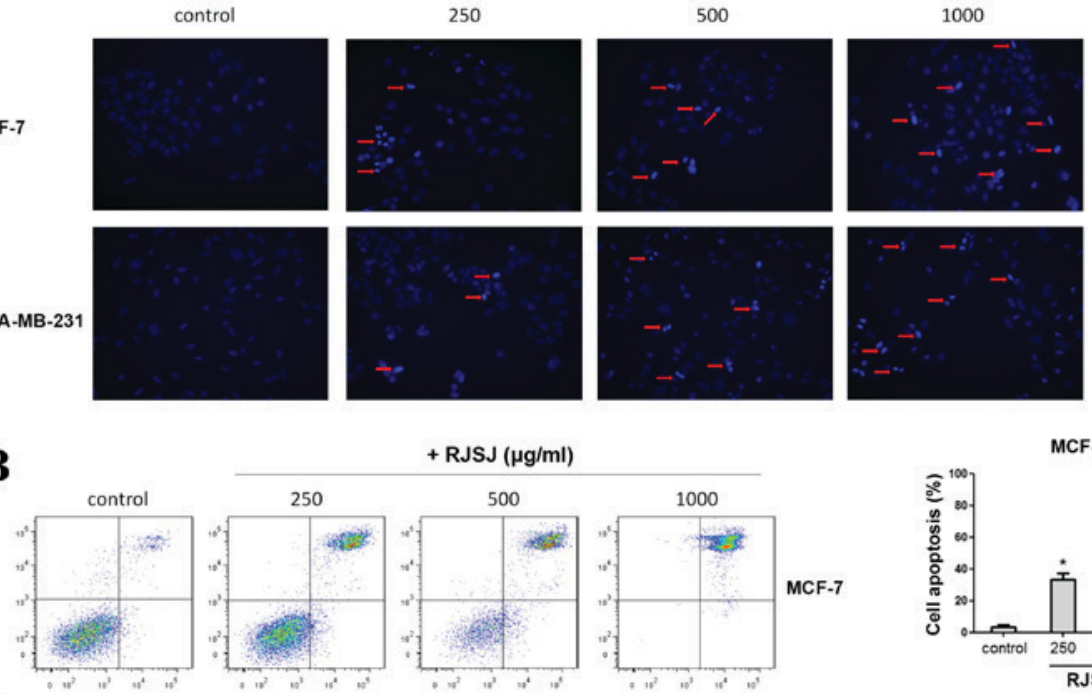

+ RJSJ ( $\mu \mathrm{g} / \mathrm{ml})$
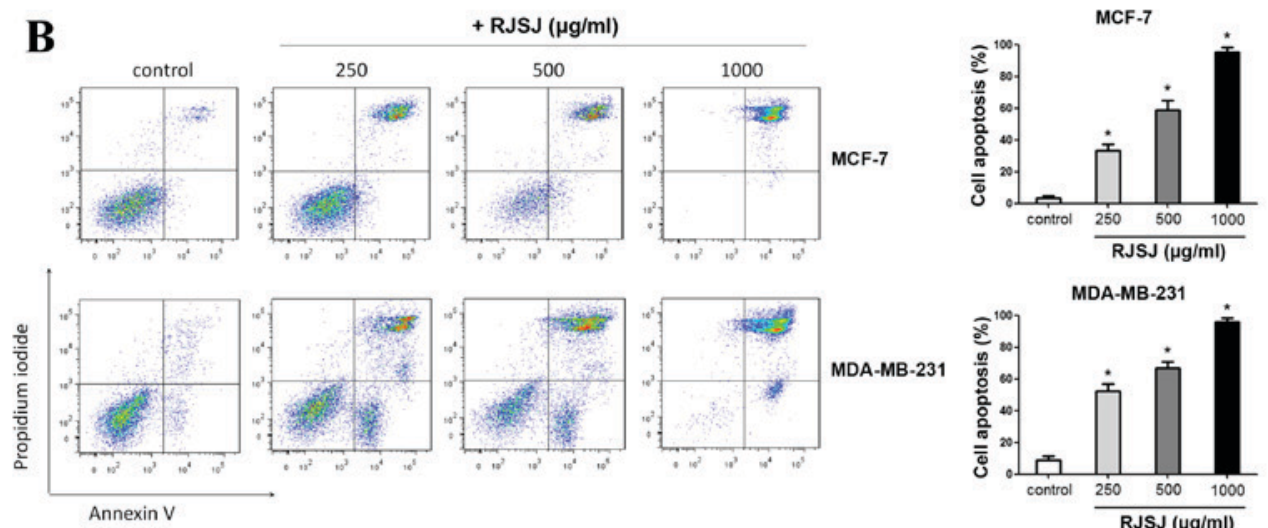

C

MCF-7
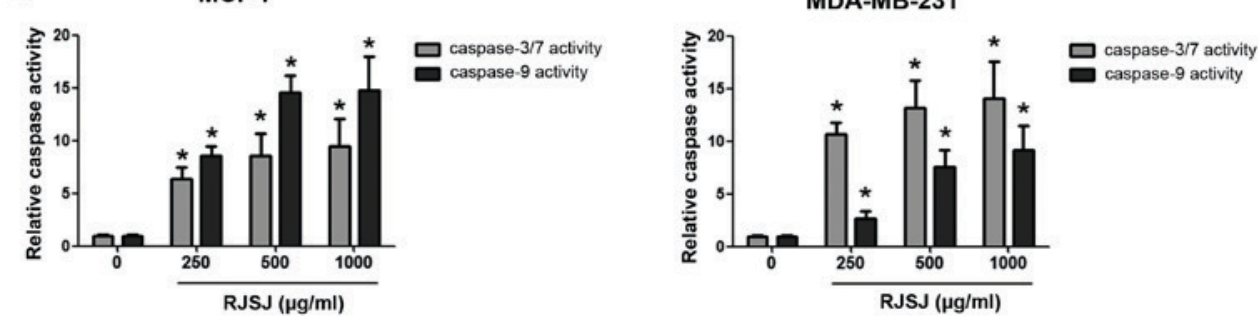

D

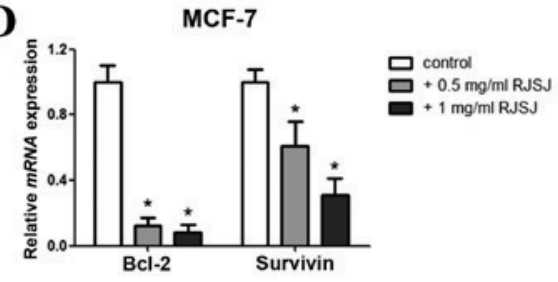

$\mathbf{E}$
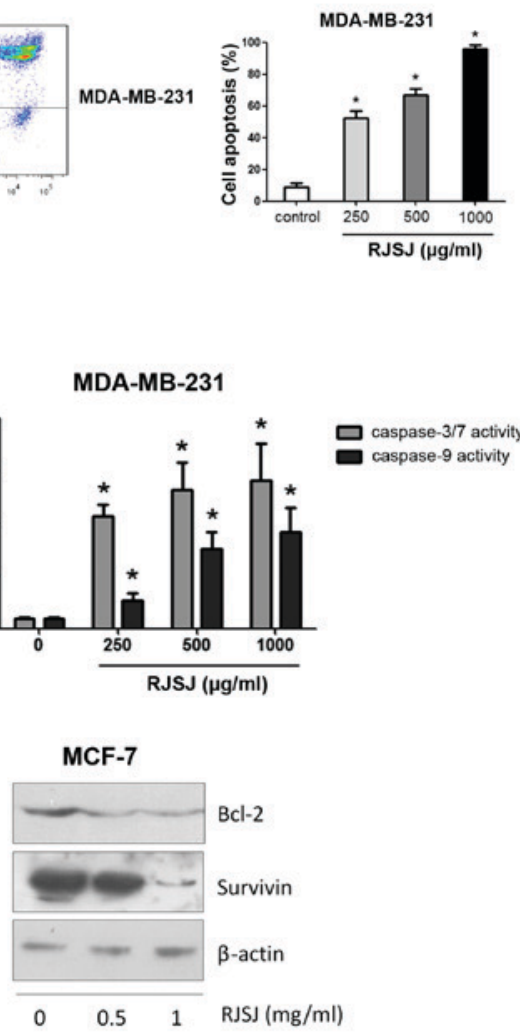

MDA-MB-231
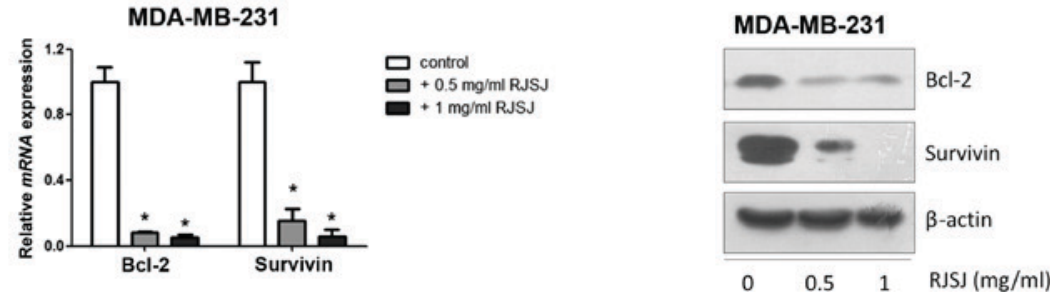

Figure 3. RJSJ induces apoptosis in breast cancer cells. (A) Representative fluorescence images of MCF-7 cells, upper panel, and MDA-MB-231 cells, lower panel, stained with Hoechst 33258 subsequent to treatment with RJSJ for $24 \mathrm{~h}$; red arrows indicate cell shrinkage and nuclear fragmentation (magnification, x200). (B) Cells were treated with RJSJ at different concentrations for $48 \mathrm{~h}$. Annexin V/propidium iodide staining was detected with flow cytometry. Representative plots of 3 independent experiments are shown. Quantitative data show the average percentage of Annexin V-positive cells in early apoptosis, lower right quadrant, and late apoptosis, upper right quadrant, of 3 independent experiments, right panel. (C) Caspase-3/7 and caspase-9 activities in MCF-7, left histograms, and MDA-MB-231, right histograms, cells subsequent to RJSJ treatment. (D and E) Bcl-2 and survivin expression levels in MCF-7 and MDA-MB-231 cells determined using (D) reverse transcription-quantitative polymerase chain reaction and (E) western blot analysis subsequent to RJSJ treatment for $48 \mathrm{~h}$. Pictorial data show representative images of 3 independent experiments. Numerical data represent the mean \pm standard deviation of 3 independent experiments. "P<0.05. RJSJ, Ruanjian Sanjie; Bcl-2, B-cell lymphoma 2; mRNA, messenger RNA. 
RJSJ promotes cell apoptosis, induces caspase activity and modulates the expression of apoptosis-associated genes. Next, the present study investigated whether the cytotoxic effect of RJSJ on breast cancer cells is mediated by promoting cell apoptosis using Hoechst 33258. Following apoptosis, an increase in membrane permeability permits the dye to enter into the cell and to stain condensed chromosomes, which is an indicator of apoptosis (21). The present study observed dense blue fluorescent particles within the nucleus or cytoplasm in addition to nuclear morphological changes following $48 \mathrm{~h}$ of incubation with RJSJ in MCF-7 and MDA-MB-231 cells (Fig. 3A), indicating the activation of apoptosis. Flow cytometric Annexin V/PI analysis additionally confirmed these initial findings, as the number of apoptotic cells increased following RJSJ treatment in a dose-dependent manner (Fig. 3B) $(\mathrm{P}=0.011)$. In order to determine whether these events were accompanied by caspase activation, the activities of caspase-3/7 and -9 were measured. In accordance with the Annexin V/PI analysis, RJSJ markedly induced caspase-3/7 and caspase- 9 activities in a dose-dependent manner in the cells (Fig. 3C) $(\mathrm{P}=0.02$ for caspase-3/7 and $\mathrm{P}=0.03$ for caspase-9). As the induction of apoptotic cell death can be partly due to the alteration of pro-survival and pro-apoptotic proteins, the present study evaluated the messenger RNA levels of numerous survival and apoptotic genes. RT-qPCR and western blotting results revealed that RJSJ inhibited the expression of the pro-survival Bcl-2 and survivin proteins in MCF-7 and MDA-MB-231 cells (Fig. 3D and E). Thus, RJSJ induces caspase activity and the downregulation of the pro-survival proteins Bcl-2 and surviving, leading to apoptosis in breast cancer cells.

Administration of RJSJ in combination with doxorubicin is more effective and safer than the chemotherapeutic treatment alone in breast cancer xenografts. As the significant antitumor activity of RJSJ was observed in both breast cancer cells and EAC tumor models, it was investigated whether the antitumor efficacy of RJSJ may be maintained in a breast cancer xenograft model generated with highly metastatic MDA-MB-231 cells. As the aim of the present study was to explore whether RJSJ may be effective in advanced breast cancer, the tumor volume was monitored, and when the average tumor volume reached $\sim 400 \mathrm{~mm}^{3}$, the tumor-bearing mice were randomly divided into four groups of 6 animals/group prior to the initiation of the treatments. Tumor growth steadily progressed during the following 15 days in the control group, whereas tumor volume increased at a lower rate in the doxorubicin- and RJSJ-treated groups. Notably, the animals receiving doxorubicin alone exhibited a significant body weight loss, $20 \%$ reduction compared with that of the control group $(\mathrm{P}=0.03)$. By contrast, no significant body weight loss was observed in the animals treated with RJSJ alone $(\mathrm{P}=0.86)$. Additionally, the group receiving doxorubicin and RJSJ displayed the smallest tumors $(\mathrm{P}=0.047)$, with no additional mouse body weight loss ( $\mathrm{P}=0.76$ for combined group vs. doxorubicin group), indicating that the RJSJ-doxorubicin combination is more efficacious and better tolerated than doxorubicin alone (Fig. 4A and B). The expression of anti-apoptotic Bcl-2 and pro-survival survivin in the tumors was consistent with the in vitro results (Fig. 3D), showing a downregulation of these genes following RJSJ
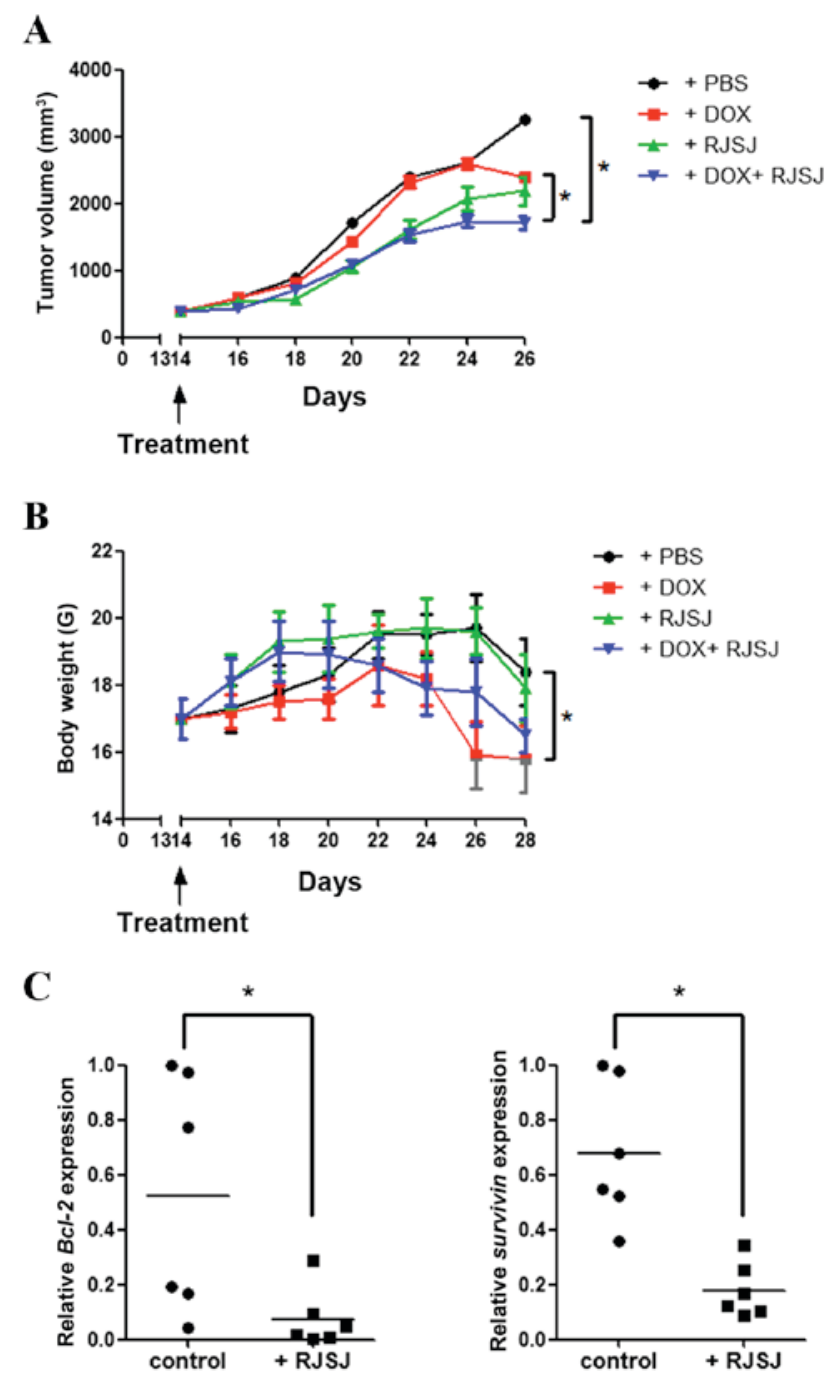

Figure 4. Antitumor activity of RJSJ in MDA-MB-231 advanced xenograft tumors. (A) Tumor size and (B) body weight of nude mice subsequent to treatment with PBS (control), DOX, RJSJ or DOX plus RJSJ. Data are presented as the mean tumor size \pm standard deviation of 6 mice per group. (C) RNA was isolated from tumors 28 days post-implantation, and Bcl-2 and survivin messenger RNA expression was determined by quantitative polymerase chain reaction. The individual tumor expression data, dots, and the mean values, lines, are indicated. ${ }^{*} \mathrm{P}<0.05$. RJSJ, Ruanjian Sanjie; Bcl-2, B-cell lymphoma 2; DOX, doxorubicin.

administration (Fig. 4C) ( $\mathrm{P}=0.03$ for $\mathrm{Bcl}-2$ expression and $\mathrm{P}=0.013$ for survivin expression). In summary, the administration of RJSJ in combination with doxorubicin is more effective in reducing tumor size than the chemotherapeutic treatment alone in mouse breast cancer xenografts.

\section{Discussion}

According to TCM, cancer is a systemic disease formed when the internal functions of the body become imbalanced (22). Folk Chinese tradition attributes breast cancer to the accumulation of toxins, heat, swelling and blood stasis in the body. This pathology is cited as 'breast rock' in the most ancient Chinese medical texts (6). More recently, pre-clinical and clinical studies have shown that TCM combined with conventional Western medicine, chemotherapy and radiotherapy, can provide effective supportive care for patients with 
cancer (23-26). TCM increases the sensitivity of chemo- and radio-therapeutics, reduces the side effects of the aforementioned therapies, and improves patient quality of life and survival time (23-26). One classical model of TCM combination therapy, called formula, has been advocated for $>2,500$ years. Formulae always consist of different herbs or minerals, as their components may act on multiple targets and show synergistic therapeutic efficacy $(27,28)$. For example, the Chinese medical formula warming and relieving cold phlegm has demonstrated an anticancer effect on hepatoma cells in vitro and on breast cancer cells in vivo $(29,30)$, whereas Xiaotan Sanjie, a traditional Chinese herbal decoction composed of 11 herbs, has been shown to be effective in gastric cancer treatment $(31,32)$. However, despite a long tradition and anecdotal evidence of cancer cures using TCM as a sole therapy (6), the key active principles in Chinese formulae and their mechanisms of action remain unknown.

In an effort to shed light on the underlying principles involved in TCM, the present study investigated the antitumor activity of RJSJ, a decoction of Pinellia ternata, Prunella vulgaris, Cremastra appendiculata and Sargassum pallidum traditionally used in China (10). Although a number of preliminary studies indicate tumor-inhibitory properties in extracts of several individual components of RJSJ (11-14), no studies on the effect of RJSJ on cancer have yet been published. The acute toxicity assays of the present study indicate that RJSJ is safe and well tolerated. However, the median lethal dose $\left(\mathrm{LD}_{50}\right)$ was not calculated, as no mortality or behavioral changes were observed at the highest dose used $(5 \mathrm{~g} / \mathrm{kg})$.

The administration of RJSJ exhibits similar efficacy in terms of tumor growth inhibition to that of 5-Fu in EAC tumor models and to doxorubicin in advanced breast cancer xenograft models. RJSJ is better tolerated than these two chemotherapeutic drugs alone, without body weight loss or toxicity due to myelosuppression. Notably, RJSJ reduces the immunosuppressive reaction of tumor-bearing mice treated by chemotherapeutic drugs and strengthens the immunocompetence of the host. Having established the excellent antiproliferative capacity of RJSJ when used in combination with standard chemotherapeutics, the present study demonstrated that the effect of RJSJ on breast cancer, both in vitro and in vivo, can be at least partially attributed to its ability to induce apoptosis. The administration of RJSJ results in the activation of caspase-3,7 and -9 , and the downregulation of survivin and Bcl-2 expression, two pro-survival proteins. These results are consistent with those from previous studies indicating that Pinellia ternate extracts induce apoptosis in human hepatoma cells (33). Prunella vulgaris also promotes apoptosis and inhibits cell proliferation in human colorectal cancer cells through targeting the signal transducer and activator of transcription 3 pathway (34), decreases Bcl-2 expression, and increases Bcl-2-like protein 4 and Bcl-2-associated death promotor expression in human pulmonary adenocarcinoma cells (35). In addition, Sargassum pallidum aqueous extract enhances antioxidant activities in rodent gastric cancer (36), whereas Cremastra appendiculata tuber extract shows potent cytotoxicity against MCF-7 and MDA-MB-231 cells in vitro (37). These data suggest that RJSJ may effectively eliminate breast cancer cells through modulating a variety of pathways in addition to apoptosis via regulating Bcl-2 and survivin expression.

In conclusion, the present study observed that the TCM RJSJ decoction shows potent antitumor activity in vitro and in vivo. RJSJ significantly induces cell apoptosis, activating caspases-3,7 and 9, and suppressing Bcl-2 and survivin expression in breast cancer cells. Additionally, considering that i.g. RJSJ treatment is well tolerated, the combination of RJSJ and chemotherapeutic drugs shows a synergistic effect in vivo, particularly in advanced breast cancer xenograft models. Additional investigation into the clinical implication of RJSJ in cancer treatment and the characterization of its active principles is required.

\section{Acknowledgements}

The present study was supported by the Chinese National Natural Sciences Foundation (grant no. 81402480), the Science and Technology Foundation of Tianjin Municipal Health Bureau (grant no. 2014KZ078) and Tianjin Medical University Cancer Institute and Hospital Foundation (grant no. 1416).

\section{References}

1. Chen W, Zheng R, Baade PD, Zhang S, Zeng H, Bray F, Jemal A, $\mathrm{Yu}$ XQ and He J: Cancer statistics in China, 2015. CA Cancer J Clin 66: 115-132, 2016.

2. Cohen SM, Mukerji R, Cai S, Damjanov I, Forrest ML and Cohen MS: Subcutaneous delivery of nanoconjugated doxorubicin and cisplatin for locally advanced breast cancer demonstrates improved efficacy and decreased toxicity at lower doses than standard systemic combination therapy in vivo. Am J Surg 202: 646-653, 2011.

3. Chen C, Sun S, Yuan JP, Wang YH, Cao TZ, Zheng HM, Jiang XQ, Gong YP, Tu Y, Yao F, et al: Characteristics of breast cancer in Central China, literature review and comparison with USA. Breast 30: 208-213, 2016.

4. Xu B, Hu X, Jiang Z, Li H, Chen J, Cui S, Li Q, Liao N, Liu D, Liu J, et al: National consensus in China on diagnosis and treatment of patients with advanced breast cancer. Ann Transl Med 3: $242,2015$.

5. Chaffer CL and Weinberg RA: A perspective on cancer cell metastasis. Science 331: 1559-1564, 2011.

6. Cohen I, Tagliaferri M and Tripathy D: Traditional Chinese medicine in the treatment of breast cancer. Semin Oncol 29: 563-574, 2002.

7. Ding X, Yang Q, Kong X, Haffty BG, Gao S and Moran MS: Radiosensitization effect of Huaier on breast cancer cells. Oncol Rep 35: 2843-2850, 2016.

8. Tao G and Balunas MJ: Current therapeutic role and medicinal potential of Scutellaria barbata in traditional Chinese medicine and Western research. J Ethnopharmacol 182: 170-180, 2016.

9. Lee YW, Chen TL, Shih YR, Tsai CL, Chang CC, Liang HH, Tseng SH, Chien SC and Wang CC: Adjunctive traditional Chinese medicine therapy improves survival in patients with advanced breast cancer: A population-based study. Cancer 120: 1338-1344, 2014.

10. Lahans T: Integrating conventional and Chinese medicine in cancer care: A clinical guide. Churchill Livingstone Elsevier, Edinburgh 2007.

11. Tan J, Qi H and Ni J: Extracts of endophytic fungus xkc-s03 from Prunella vulgaris L. Spica inhibit gastric cancer in vitro and in vivo. Oncol Lett 9: 945-949, 2015.

12. Kim SH, Huang CY, Tsai CY, Lu SY, Chiu CC and Fang K: The aqueous extract of Prunella vulgaris suppresses cell invasion and migration in human liver cancer cells by attenuating matrix metalloproteinases. Am J Chin Med 40: 643-656, 2012.

13. Kurt O, Ozdal-Kurt F, Tuğlu MI and Akçora CM: The cytotoxic, neurotoxic, apoptotic and antiproliferative activities of extracts of some marine algae on the MCF-7 cell line. Biotech Histochem 89: 568-576, 2014. 
14. Aravindan S, Ramraj SK, Somasundaram ST, Herman TS and Aravindan N: Polyphenols from marine brown algae target radiotherapy-coordinated EMT and stemness-maintenance in residual pancreatic cancer. Stem Cell Res Ther 6: 182, 2015.

15. National Research Council (US) Committee for the Update of the Guide for the Care and Use of Laboratory Animals, Guide for the Care and Use of Laboratory Animals, 8th edition. Washington (DC): National Academies Press (US); 2011.

16. Alam B, Majumder R, Akter S and Lee SH: Piper betle extracts exhibit antitumor activity by augmenting antioxidant potential. Oncol Lett 9: 863-868, 2015.

17. Hu Y, Guo R, Wei J, Zhou Y, Ji W, Liu J, Zhi X and Zhang J: Effects of PI3K inhibitor NVP-BKM120 on overcoming drug resistance and eliminating cancer stem cells in human breast cancer cells. Cell Death Dis 6: e2020, 2015.

18. Hu Y, Li K, Asaduzzaman M, Cuella R, Shi H, Raguz S, Coombes RC, Zhou Y and Yagüe E: miR-106b 25 cluster regulates multidrug resistance in an $\mathrm{ABC}$ transporter-independent manner via downregulation of EP300. Oncol Rep 35: 1170-1178, 2016.

19. Livak KJ and Schmittgen TD: Analysis of relative gene expression data using real-time quantitative PCR and the 2(-Delta Delta C(T)) method. Methods 25: 402-408, 2001.

20. Peng M, Ding Y, Yu L, Deng Y, Lai W, Hu Y, Zhang H, Wu X, Fan H, Ding H, et al: Tegafur substitution for 5-Fu in combination with actinomycin D to treat gestational trophoblastic neoplasm. PLoS One 10: e0143531, 2015.

21. Sun HY, Liu BB, Hu JY, Xu LJ, Chan SW, Chan CO, Mok DK, Zhang DM, Ye WC and Chen SB: Novel cycloartane triterpenoid from Cimicifuga foetida (Sheng ma) induces mitochondrial apoptosis via inhibiting Raf/MEK/ERK pathway and Akt phosphorylation in human breast carcinoma MCF-7 cells. Chin Med 11: 1, 2016

22. Chung VC, Wu X, Lu P, Hui EP, Zhang Y, Zhang AL, Lau AY, Zhao J, Fan M, Ziea ET, et al: Chinese herbal medicine for symptom management in cancer palliative care: Systematic review and meta-analysis. Medicine (Baltimore) 95: e2793, 2016.

23. Efferth T, Kahl S, Paulus K, Adams M, Rauh R, Boechzelt H, Hao X, Kaina B and Bauer R: Phytochemistry and pharmacogenomics of natural products derived from traditional Chinese medicine and Chinese materia medica with activity against tumor cells. Mol Cancer Ther 7: 152-161, 2008.

24. Man YN, Liu XH and Wu XZ: Chinese medicine herbal treatment based on syndrome differentiation improves the overall survival of patients with unresectable hepatocellular carcinoma. Chin J Integr Med 21: 49-57, 2015.

25. Konkimalla VB and Efferth T: Evidence-based Chinese medicine for cancer therapy. J Ethnopharmacol 116: 207-210, 2008.
26. Lam W, Bussom S, Guan F, Jiang Z, Zhang W, Gullen EA, Liu SH and Cheng YC: The four-herb Chinese medicine PHY906 reduces chemotherapy-induced gastrointestinal toxicity. Sci Transl Med 2: 45ra59, 2010.

27. Qi F, Li A, Inagaki Y, Gao J, Li J, Kokudo N, Li XK and Tang W: Chinese herbal medicines as adjuvant treatment during chemoor radio-therapy for cancer. Biosci Trends 4: 297-307, 2010

28. Cao R, Zhang H, Guo J, Liu XH, Liu C, Zhu CH and Wu XZ: A novel pharmacological method to study the Chinese medicinal formula Hua-Zheng-Hui-Sheng-Dan. Evid Based Complement Alternat Med 2015: 436807, 2015.

29. Yan ZC, Chen D, Wu XZ, Xie GR, Ba Y and Yan Z: Effects of aqueous extracts of Aconitum carmichaeli, Rhizoma bolbostemmatis, Phytolacca acinosa, Panax notoginseng and Gekko swinhonis Guenther on Bel-7402 cells. World J Gastroenterol 13: 2743-2746, 2007.

30. Wang XL, Ma F and Wu XZ: Anticancer effects of 5-fluorouracil combined with warming and relieving cold phlegm formula on human breast cancer. Chin J Integr Med 18: 599-604, 2012.

31. Shi J and Wei PK: Xiaotan Sanjie decoction inhibits interleukin-8-induced metastatic potency in gastric cancer. World J Gastroenterol 21: 1479-1487, 2015.

32. Yan B, Liu L, Zhao Y, Xiu LJ, Sun DZ, Liu X, Lu Y, Shi J, Zhang YC, Li YJ, et al: Xiaotan Sanjie decoction attenuates tumor angiogenesis by manipulating Notch-1-regulated proliferation of gastric cancer stem-like cells. World J Gastroenterol 20: 13105-13118, 2014

33. Zhou W, Gao Y, Xu S, Yang Z and Xu T: Purification of a mannose-binding lectin Pinellia ternata agglutinin and its induction of apoptosis in Bel-7404 cells. Protein Expr Purif 93: 11-17, 2014.

34. Lin W, Zheng L, Zhuang Q, Zhao J, Cao Z, Zeng J, Lin S, Xu W and Peng J: Spica prunellae promotes cancer cell apoptosis, inhibits cell proliferation and tumor angiogenesis in a mouse model of colorectal cancer via suppression of stat3 pathway. BMC Complement Altern Med 13: 144, 2013.

35. Feng L, Au-Yeung W, Xu YH, Wang SS, Zhu Q and Xiang P: Oleanolic acid from Prunella vulgaris L. induces SPC-A-1 cell line apoptosis via regulation of $\mathrm{Bax}, \mathrm{Bad}$ and $\mathrm{Bcl}-2$ expression. Asian Pac J Cancer Prev 12: 403-408, 2011.

36. Zhang RL, Luo WD, Bi TN and Zhou SK: Evaluation of antioxidant and immunity-enhancing activities of Sargassum pallidum aqueous extract in gastric cancer rats. Molecules 17: 8419-8429, 2012.

37. Liu L, Li J, Zeng KW, Jiang Y and Tu PF: Five new benzylphenanthrenes from Cremastra appendiculata. Fitoterapia 103: 27-32, 2015. 